\title{
Gestión Academica y Desempeño Docente, según los estudiantes de una universidad privada en Lima, Perú
}

Daniel Víctor Surco Salinas ${ }^{1}$

RECIBIDO:22/11/2017 ACEPTADO: 04/05/2018

\begin{abstract}
RESUMEN
Actualmente, cabe resaltar que la Gestión Académica (GA) debería ser un criterio clave para la gestion de las instituciones educativas y asimismo el Desempeño Docente (DD) estar enmarcado en las metas institucionales de dichas organizaciones. El objetivo de la investigación fue determinar el grado de relación que existe entre la GA y el $\mathrm{DD}$, según la percepción de los estudiantes de una universidad privada. El Alfa de Cronbach presentó índices aceptables de 0.934 para la GA y 0.946 para el DD. En la validez de los instrumentos, se alcanzó 0.823 para la GA y 0.814 para el DD. Los resultados de la investigación han puesto en evidencia que existe una correlación Spearman (rho) moderada y positiva entre la GA y el DD con un valor de 0.629 , mientras que la correlación Spearman entre las dimensiones de la GA y el DD varía entre los valores 0.432 y 0.608 ,que son significativos.
\end{abstract}

Palabras-claves: Gestión Académica; Desempeño Docente.

Academic Management and Teaching Performance, ACCORDING TO THE STUDENTS OF A PRIVATE UNIVERSITY IN Lima, Peru

\section{ABSTRACT}

Currently, it should be noted that the Academic Management $(G A)$ should be a key criterion for the management of educational institutions and also the Teaching Performance (DD) be framed in the institutional goals of these organizations. The objective of the research was to determine the degree of relationship that exists between the GA and the $\mathrm{DD}$, according to the perception of the students of a private university. Cronbach's Alpha presented acceptable indexes of 0.934 for $\mathrm{GA}$ and 0.946 for $\mathrm{DD}$. In the validity of the instruments, 0.823 was reached for $G A$ and 0.814 for $\mathrm{DD}$. The results of the investigation have shown that there is a moderate and positive Spearman (rho) correlation between the $G A$ and DD with a value of 0.629 , while the Spearman correlation between the GA and the $\mathrm{DD}$ dimensions varies between the values 0.432 and 0.608 , which are significant.

Keywords: Academic Management; Teaching Performance.

\section{INTRODUCCIÓN}

Actualmente, es muy importante el rol que tiene la gestión en el sector de la educación a nivel superior, ya que se busca optimizar la productividad en dicho sector, basándose en la eficiencia y eficacia, que al mismo tiempo son indicadores del mejoramiento continuo que se debe transmitir a la calidad de la educación. Asimismo cuando la gestión se orienta a la obtención de resultados en la educación significa poner en el primer plano del sistema, a la institución educativa, responsable de la formación, y desde este punto establecer los procesos, insumos, actividades y productos necesarios para alcanzar adecuados índices de calidad de los aprendizajes que se persiguen. Y específicamente cuando se habla de la GA. Al respecto, Martín, Perdomo y Segredo (2013), exponen:

De ahí que, en la gestión académica se realicen un conjunto de actividades encaminadas a facilitar la transformación de las condiciones institucionales con espíritu de renovación e investigación, en búsqueda de soluciones a los problemas o necesidades identificadas durante el desarrollo del proceso enseñanza aprendizaje. (p.290)

Por otra parte, en pleno siglo XXI el DD a nivel universitario es importante en las instituciones educativas del sector, ya que los nuevos cambios sociales traen nuevos desafíos a la enseñanza universitaria y exigen al docente a ser innovador tanto en el aspecto pedagógico y tecnológico. Considerando un valioso aporte, Almuiñas y Galarza (2013), mencionaron:

(...) La imagen más actual del docente universitario es la de un profesional que toma decisiones, investigador de su aula y fuera de ella, alguien que resuelve los problemas que se le presentan con una actitud ética, humanista y solidaria, que opta por determinadas soluciones ante un dilema y se preocupa y ocupa constantemente por su superación, entre otros aspectos. Su concreción en la práctica sólo es posi-

\footnotetext{
1 Ingeniero Industrial

Diplomado en Gestion de Negocios

Diplomado en Comercio Exterior

Docente Universitario

Universidad Privada Telesup

Facultad de Ciencias Administrativas y Contables

Escuela Académico Profesional de Administración de Empresas

http://orcid.org/0000-0001-9883-5249 - surco@telesup.net
} 
ble cuando existan espacios y condiciones para que el mismo aplique exitosamente las capacidades adquiridas, es decir, para favorecer que sea competente. (p.32)

Precisamente, hoy en día las organizaciones empresariales ya sea en cualesquiera de los sectores económicos de un país, hacen bastante énfasis en los resultados que esperan obtener durante una gestión en particular. Para que se puedan alcanzar dichos resultados es necesario contar con una diversidad de recursos de todo tipo. Y cuando se considera el sector educativo es de sapiencia universal que el personal docente es uno de los principales recursos con los que cuenta una institución educativa, sobre todo una universidad.

Loureiro y Míguez (2006) citados en Loureiro, Míguez y Otegui (2016), mencionaron:

En la evaluación docente, el objeto de estudio es el desempeño docente en la enseñanza y debe caracterizarse por ser: integral, evaluando diversos aspectos de la función docente; permanente, ya que la evaluación podrá identificar fortalezas y dificultades sólo cuando es continua; participativa, involucrando a los diversos actores de la institución, y contextual, en el sentido de que sea acorde a la realidad de la institución, sistematizando y extendiendo aquellas experiencias que ya se han desarrollado. Se considera que la evaluación docente debe permitir una retroalimentación, ya que debe dar información a los docentes sobre sus logros y sus dificultades y al resto de la institución sobre la enseñanza; la toma de decisiones, los resultados de la evaluación docente deben ser un insumo para tomar decisiones orientadas a mejorar el proceso de enseñanza y la identificación de necesidades de formación, desarrollando las estrategias más adecuadas para satisfacerlas. (p. 56)

Por lo tanto, al tratar esta variable en la educación superior hay que considerar básicamente que el rol del docente debería estar enmarcado con las metas institucionales de las organizaciones educativas universitarias a fin de alcanzar la eficacia en la calidad del proceso enseñanza - aprendizaje. En relación a esta variable, Sánchez y Solar (2007), mencionaron lo siguiente:

El desempeño docente abarca funciones, tareas y roles de los docentes universitarios, que se analizan desde su propia concepción (creencias, pensamientos, percepciones, teorías implícitas) y de poseer sólida base de conocimientos disciplinarios, profesionales y pedagógicos. La enseñanza es un proceso multidimensional y complejo, por ello las acciones que involucran el desempeño son dinámicas y deben estar en constante adaptación a los cambios y demandas del sistema social. El docente que tiene un conocimiento más profundo y una conciencia crítica sobre los diferentes componentes y variables de la enseñanza - aprendizaje está mejor preparado para realizar juicios y tomar decisiones apropiadas sobre este proceso. (p. 148)

Asimismo, a través de la investigación se consideró la aplicación de los enfoques teóricos que se manejan actualmente en cuanto a la concepción de las variables investigadas: GA y DD. Así como la conceptualización de las dimensiones respectivas, tales como diseño curricular, prácticas pedagógicas, seguimiento académico, habilidades comunicativas, etc. Desde el punto de vista metodológico, para lograr los objetivos de esta investigación se utilizó las técnicas de investigación como el cuestionario y su procesamiento respectivo con el software apropiado (SPSS) que permitió medir el grado de relación existente entre la GA y el DD en una universidad privada de Lima.

\section{METODOLOGIA}

\subsection{Diseño}

La investigación tuvo un diseño no experimental de corte transversal y correlacional. Hernández, Fernández y Baptista (2010) sostuvieron que "la investigación no experimental comprende los estudios que se realizan sin la manipulación deliberada de variables y en los que sólo se observan los fenómenos en su ambiente natural para después analizarlos. (...)Los diseños de investigación transaccional o transversal recolectan datos en un solo momento, en un tiempo único. Su propósito es describir variables y analizar su incidencia e interrelación en un momento dado".

\subsection{Participantes}

El universo para el estudio de investigación estuvo conformado por los estudiantes de la modalidad presencial comprendidos entre el tercer ciclo al décimo ciclo de la Facultad de Ciencias Administrativas y Contables de una universidad privada en Lima, siendo la cantidad de 650 estudiantes matriculados para el semestre 2017-I. La muestra representativa para el caso de los estudiantes fue de 242 estudiantes. Para la determinación de la muestra se consideró estudiantes de cada una de las carreras profesionales de la Facultad, seleccionándose por muestreo estratificado, donde todos los alumnos 
tuvieron las mismas oportunidades para ser elegidos. En la muestra se tuvo como edad mínima 18 años y como edad máxima 26 años. De la misma forma, la edad promedio es 22.28 años, la edad con mayor frecuencia es 23 años y además la mitad de los estudiantes tienen como máximo 22.50 años. La muestra se compone por un $31.8 \%$ de varones y un $68.2 \%$ de mujeres.

\subsection{Instrumentos de medición}

Para la recolección de datos se utilizó como técnica las encuestas, las cuales se aplicaron en un solo instante considerando la muestra respectiva, siendo elaboradas basándose en la diversa literatura expuesta en el marco teórico. Para la recolección de datos de la variable GA se aplicó la encuesta denominada "Cuestionario relativo a los procesos de Gestión Académica”, elaborado por el autor de la presente investigación, que permitió inferir en qué medida se manifiestan los estudiantes por el nivel de aceptación de la GA. Se consideró para la elaboración del instrumento 5 dimensiones, con un total de 31 ítems, a base de la escala de Likert y estableciéndose cinco alternativas de respuesta: $1=\mathrm{No} /$ Rara vez, $2=$ Casi nada, $3=$ Parcialmente, $4=$ En Gran parte, 5= Totalmente. Con respecto a la recolección de datos de la variable DD se aplicó la encuesta denominada "Cuestionario relativo a las áreas del Desempeño Docente", elaborada por el autor de la presente investigación, que permitió in- ferir en qué medida se manifiestan los estudiantes por el nivel de aceptación del DD. Para la construcción del instrumento se consideró 4 dimensiones, con un total de 27 ítems, a base de la escala de Likert y estableciéndose cinco alternativas de respuesta: 1= Nunca, 2= Casi nunca, 3= Algunas veces, $4=$ Casi siempre, $5=$ Siempre. Ver Tabla $N^{\circ} 1$.

A fin de establecer la consistencia interna de los instrumentos mediante los coeficientes de estimación de confiabilidad respectivos, se aplicó la prueba denominada Alfa de Cronbach a un grupo piloto de 25 estudiantes, obteniéndose para la GA una consistencia interna Alfa $=0.934$ ( bueno) $y$, para el DD una consistencia interna Alfa $=0.946$ (bueno).

Además, con la finalidad de establecer la validez del contenido, que determina el grado en el que la medición representa la variable medida, los instrumentos se sometieron al Juicio de Expertos (Método Delphi). Los instrumentos fueron evaluados por 4 catedráticos competentes de la Facultad de Educación de la UNMSM, cuyos resultados se muestran en las tablas $\mathrm{N}^{\circ} 2$ y $\mathrm{N}^{\circ} 3$. Y en función de una escala evaluativa de 5 niveles desde Deficiente (mínimo) hasta Excelente (máximo), con diez aspectos con sus respectivos criterios, el instrumento relacionado a la GA alcanzó el $82.25 \%$ de validez (Excelente) mientras el instrumento relacionado al DD alcanzó el $81.38 \%$ de validez (Excelente).

Tabla $\mathbf{N}^{\circ}$ 1. Variables, Dimensiones e Indicadores de la investigación

\begin{tabular}{|c|c|c|c|}
\hline \multicolumn{2}{|r|}{ GESTION ACADEMICA } & \multicolumn{2}{|c|}{ DESEMPEÑO DOCENTE } \\
\hline DIMENSIONES & INDICADORES & DIMENSIONES & INDICADORES \\
\hline Diseño Curricular & $\begin{array}{ll}\text { - } & \text { Plan de estudios } \\
\text { - } & \text { Enfoque metodológico } \\
\text { - } & \text { Programación académica } \\
\text { - } & \text { Jornada universitaria } \\
\text { - } & \text { Evaluación } \\
\end{array}$ & Enseñanza y Aprendizaje & $\begin{array}{l}\text { - Dominio de la Asignatura } \\
\text { - Motivación para el apren- } \\
\text { dizaje } \\
\text { - Nivel Cultural }\end{array}$ \\
\hline Prácticas Pedagógicas & $\begin{array}{l}\text { - Estandarización de opciones didácticas } \\
\text { - Estrategias para las tareas universitarias } \\
\text { - Uso de los tiempos para el aprendizaje }\end{array}$ & $\begin{array}{l}\text { Estrategias Pedagógicas y } \\
\text { Metodológicas }\end{array}$ & $\begin{array}{l}\text { - Estrategias cognitivas } \\
\text { - Métodos y técnicas didác- } \\
\text { ticas }\end{array}$ \\
\hline Gestión de Aula & 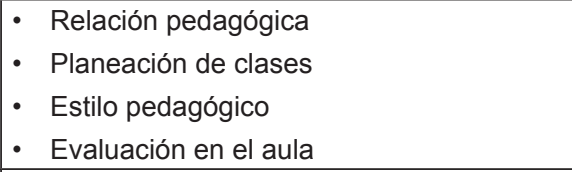 & Habilidades Comunicativas & - Comunicación eficaz \\
\hline Seguimiento Académico & $\begin{array}{l}\text { - Seguimiento a los resultados académicos } \\
\text { - Expectativa académica-profesional } \\
\text { - Seguimiento a la asistencia } \\
\text { - Seguimiento a los egresados }\end{array}$ & Actitud hacia la Docencia & $\begin{array}{l}\text { - Ética profesional } \\
\text { - Control conductual }\end{array}$ \\
\hline Innovación y Aprendizaje & - Inversión en la investigación & & \\
\hline
\end{tabular}

Fuente: Elaboración propia. 
Tabla $N^{\circ}$ 2. Validez de Instrumento: Cuestionario relativo a los procesos de Gestión Académica

\begin{tabular}{|c|c|c|c|c|c|}
\hline \multirow{2}{*}{$\begin{array}{l}\text { Aspectos } \\
\text { evaluados }\end{array}$} & \multicolumn{4}{|c|}{ Experto } & \multirow{2}{*}{$\begin{array}{c}\text { Promedio } \\
(\%)\end{array}$} \\
\hline & A & B & C & D & \\
\hline Claridad & 78 & 78 & 83 & 78 & 79.25 \\
\hline Objetividad & 83 & 78 & 83 & 88 & 83.00 \\
\hline Actualidad & 83 & 78 & 78 & 88 & 81.75 \\
\hline Organización & 78 & 73 & 78 & 93 & 80.50 \\
\hline Suficiencia & 83 & 78 & 83 & 93 & 84.25 \\
\hline Intencionalidad & 83 & 78 & 83 & 88 & 83.00 \\
\hline Consistencia & 78 & 78 & 78 & 88 & 80.50 \\
\hline Coherencia & 83 & 78 & 78 & 93 & 83.00 \\
\hline Metodología & 78 & 78 & 83 & 93 & 83.00 \\
\hline Oportunidad & 83 & 78 & 83 & 93 & 84.25 \\
\hline Promedio (\%) & 81.00 & 77.50 & 81.00 & 89.50 & 82.25 \\
\hline
\end{tabular}

Fuente: Elaboración propia.

Tabla $\mathbf{N}^{\circ}$ 3. Validez de Instrumento: Cuestionario relativo a las áreas del Desempeño Docente

\begin{tabular}{|c|c|c|c|c|c|}
\hline \multirow{2}{*}{$\begin{array}{l}\text { Aspectos } \\
\text { evaluados }\end{array}$} & \multicolumn{4}{|c|}{ Experto } & \multirow{2}{*}{$\begin{array}{c}\text { Promedio } \\
(\%)\end{array}$} \\
\hline & A & B & C & D & \\
\hline Claridad & 78 & 78 & 83 & 83 & 80.50 \\
\hline Objetividad & 78 & 78 & 83 & 83 & 80.50 \\
\hline Actualidad & 83 & 78 & 83 & 88 & 83.00 \\
\hline Organización & 83 & 73 & 83 & 78 & 79.25 \\
\hline Suficiencia & 83 & 73 & 83 & 88 & 81.75 \\
\hline Intencionalidad & 83 & 73 & 78 & 88 & 80.50 \\
\hline Consistencia & 78 & 78 & 83 & 88 & 81.75 \\
\hline Coherencia & 78 & 78 & 83 & 88 & 81.75 \\
\hline Metodología & 83 & 78 & 78 & 88 & 81.75 \\
\hline Oportunidad & 83 & 78 & 83 & 88 & 83.00 \\
\hline Promedio (\%) & 81.00 & 76.50 & 82.00 & 86.00 & 81.38 \\
\hline
\end{tabular}

Fuente: Elaboración propia.

\subsection{Procedimiento}

En este punto la recolección de datos de la realidad permitió disponer de evidencia empírica que confirme o contradiga la hipótesis planteada. Asimismo con la aplicación de los instrumentos previstos se obtuvo los resultados respectivos que previamente fueron sometidos a un análisis de depuración, crítica y consolidación.Ambos instrumentos se aplicaron en un tiempo de dos semanas. Además, su aplicación fue de manera simultánea y anónima, considerándose 30 minutos promedio para su realización y asimismo fue inspeccionado por una persona capacitada para dicha labor. En consecuencia todo esto previo consentimiento informado de los involucrados. Luego se procedió al análisis esta- dístico codificándose cada uno de los instrumentos para procesarlos mediante el programa estadístico SPSS 23.0, a fin de comprobar la relación entre las variables $\mathrm{GA}$ y $\mathrm{DD}$.

\section{RESULTADOS Y DISCUSIÓN}

Para la valoración de la GA se diseñó una escala valorativa, propuesta por el autor, mostrada en la tabla $N^{\circ} 4$. Por otra parte, para la variable $D D$ se elaboró una escala valorativa, adaptada según Association of American Schools in South America, Manual OSAC / AASSA (2010), mostrada en la tabla $\mathrm{N}^{\circ} 5$.

Tabla $\mathbf{N}^{\circ}$ 4. Escala de valoración de la Gestión Académica

\begin{tabular}{|l|c|}
\hline Valoración Cualitativa & Valoración Cuantitativa \\
\hline Excelente & $146-155$ \\
\hline Bueno & $120-145$ \\
\hline Aceptable & $85-119$ \\
\hline Regular & $50-84$ \\
\hline Inaceptable & $00-49$ \\
\hline
\end{tabular}

Fuente: Elaboración propia.

Tabla $N^{\circ}$ 5. Escala de Valoración del Desempeño Docente

\begin{tabular}{|l|c|}
\hline Valoración Cualitativa & Valoración Cuantitativa \\
\hline Ejemplar & $121-135$ \\
\hline Muy competente & $76-120$ \\
\hline En desarrollo & $36-75$ \\
\hline Inaceptable & $00-35$ \\
\hline
\end{tabular}

Fuente: Elaboración propia.

En función de la información procesada con el software estadístico respectivo, relacionada a los cuestionarios aplicados a la muestra en estudio, las tablas $\mathrm{N}^{\circ} 6$ y $\mathrm{N}^{\circ} 7$ presentan en resumen la frecuencia y porcentaje de estos resultados:

Tabla $\mathbf{N}^{\circ}$ 6. Frecuencia y porcentaje de resultados Gestión Académica

\begin{tabular}{|l|c|c|}
\hline Categoría & Frecuencia Absoluta & Porcentaje (\%) \\
\hline Excelente & 0 & 0.0 \\
\hline Bueno & 4 & 1.7 \\
\hline Aceptable & 59 & 24.4 \\
\hline Regular & 161 & 66.5 \\
\hline Inaceptable & 18 & 7.4 \\
\hline & 242 & 100.0 \\
\hline
\end{tabular}

Fuente: Elaboración propia. 
Tabla $\mathbf{N}^{\circ}$ 7. Frecuencia y porcentaje de resultados Desempeño Docente

\begin{tabular}{|l|c|c|}
\hline Categoría & Frecuencia Absoluta & Porcentaje (\%) \\
\hline Ejemplar & 6 & 2.5 \\
\hline Muy competente & 166 & 68.6 \\
\hline En desarrollo & 70 & 28.9 \\
\hline Inaceptable & 0 & 0.0 \\
\hline & 242 & 100.0 \\
\hline
\end{tabular}

Fuente: Elaboración propia.

En la tabla $\mathrm{N}^{\circ} 6$, es de notoriedad que el $66.5 \%$ (161 estudiantes) considera que la GA en la Facultad alcanza una valoración de "Regular". Mientras que de lo observado en la tabla $\mathrm{N}^{\circ} 7$, el $68.6 \%$ (166 estudiantes) opina que el DD logrado por los docentes de la Facultad se ubica en la categoría "Muy competente".

A fin de evaluar la relación de las variables en estudio, se realizó el tratamiento estadístico respectivo, para lo cual se empleó la Estadística Descriptiva e Inferencial, efectuando el análisis estadístico de asunción de normalidad: Prueba Kolmogorov-Smirnov (K-S de una muestra) y la Prueba Estadística No Paramétrica de Correlación Spearman Rho para dos variables. El nivel de significancia utilizado fue $\alpha=0.05$, y los resultados se muestran en la tabla $\mathrm{N}^{\circ} 8$.

Tabla N ${ }^{\circ}$ 8. Prueba de Normalidad KolmogorovSmirnov - Gestión Académica y Desempeño Docente

\begin{tabular}{|l|c|}
\hline Variables y Dimensiones & $\begin{array}{c}\text { Kolmogorov - Smirnov } \\
\text { SIG. }\end{array}$ \\
\hline Desempeño Docente & 0.061 \\
\hline Gestión Académica & 0.010 \\
\hline Diseño Curricular & 0.008 \\
\hline Prácticas Pedagógicas & 0.000 \\
\hline Gestión en el Aula & 0.000 \\
\hline Seguimiento Académico & 0.000 \\
\hline Innovación y Aprendizaje & 0.001 \\
\hline
\end{tabular}

Fuente: Elaboración propia.
De la tabla $N^{\circ} 8$, se deduce que la variable DD posee una distribución normal ya que $p>0.05$ mientras que la variable GA y sus dimensiones no poseen una distribución normal ya que $p<0.05$.

Continuando con el análisis estadístico, se buscó demostrar la hipótesis general $\left(\mathrm{HG}_{1}\right)$ que manifiesta la correlación existente entre las variables GA y DD, por lo que se realizó la Prueba no Paramétrica de Correlación de Spearman. Los resultados se muestran en el cuadro $\mathrm{N}^{\circ} 1$.

Realizada la prueba de correlación respectiva se determinó la relación lineal, moderada y positiva entre las dos variables y cuyo valor fue de 0.629 . De esta manera, se demuestra la hipótesis general previamente mencionada, que plantea una relación significativa entre la GA y el DD. Mientras que para el DD y las dimensiones de la GA, los valores de correlación se encuentran entre 0.432 y 0.608 , los cuales son significativos.

Al respecto, a la hipótesis general de las variables GA y DD, coinciden Correa, Correa y Álvarez (2009), indicando:

La gestión académica permite además valorar la profesionalidad del docente, esto es, el reconocimiento a su formación, a su nivel de competencia, a su relación pedagógica con los alumnos y su capacidad de interacción y de trabajo en equipo con directivos, docentes, padres de familia y otros actores de la comunidad educativa. Así mismo, reconoce la profesión del maestro como una práctica social que cobra sentido en el acto educativo, es decir, en el proceso enseñanza aprendizaje como quehacer fundacional de la misión académico pedagógica del maestro. (p.16)

Por otra parte, se afirma que existe una correlación significativa (moderada y positiva) entre la dimensión Diseño Curricular y la variable DD, obteniéndose un valor Rho $=0.527$ que equivale al $52.7 \%$ de explicación, con lo cual se confirma que al mejorar el Diseño Curricular mejora el DD. Entonces, se enfatiza lo señalado por Alvarado,

Cuadro $N^{\circ}$ 1. Prueba de Correlación de Spearman - Variables y Dimensiones

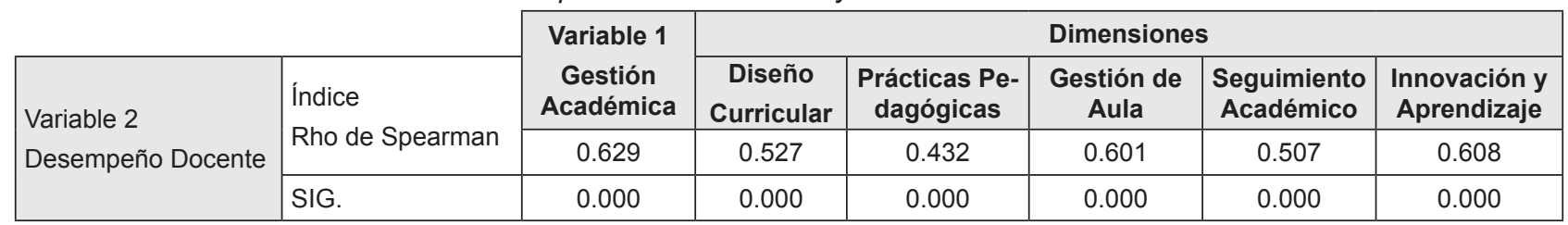

Fuente: Elaboración propia $(\mathrm{p}<0.05)$. 
Cárcamo, García y Mella (2008, p.103), cuando exponen que:

Una segunda premisa sustentadora del diseño curricular corresponde a la centralidad en los resultados de aprendizaje del estudiante, foco actual de la educación en todos los niveles. Esta focalización se asocia a otros aspectos que permiten evaluar también la gestión de la calidad de la formación que se realiza a través del seguimiento y el subsecuente mejoramiento de los índices de eficiencia educativa (mejorar retención y titulación, disminuir deserción), por una parte; por otra, se asocia asimismo a la evaluación del aprendizaje del estudiante, la que le permite realizar una retroalimentación sobre su progreso, autoevaluación y reflexión sobre sus estrategias y métodos para aprender, logrados a través del desarrollo de su autonomía académica, como también a través de los instrumentos evaluativos cuantitativos y cualitativos - instalados por el docente en su praxis.

Al mismo tiempo, se observa una correlación significativa (aceptable y positiva) entre la dimensión Prácticas Pedagógicas y la variable DD, consiguiéndose $\mathrm{Rho}=0.432$ que equivale al $43.2 \%$ de explicación: permitiendo afirmar que si mejora la dimensión Prácticas Pedagógicas entonces mejora la variable DD.

En relación a lo mencionado en el párrafo anterior, De Escallón, Porter y Richler (2013, p.63) sostuvieron que "un buen docente debe tener claridad sobre qué es la educación, una visión de educación para la diversidad y una claridad de cómo genera sus prácticas pedagógicas sobre lo que deben aprender sus estudiantes, y cómo debe enseñar y evaluar los aprendizajes".

De la misma forma, se corrobora que existe una correlación significativa (moderada y positiva) entre la dimensión Gestión de Aula y la variable DD, obteniéndose un valor Rho $=0.601$ que equivale al $60.1 \%$ de explicación, con lo cual se confirma que al mejorar la Gestión de Aula mejora el DD. Al respecto Torrego (2008) expresa:

La forma de ejercer el liderazgo es definitiva para explicar la capacidad del profesorado para controlar la disciplina en el aula. A partir de los estudios realizados sobre los estilos de interacción de los profesores con sus alumnos (Brekelman, Levy y Rodríguez, 1993), se han intentado analizar los comportamientos docentes clasificándolos conforme a dos parámetros; la proxi- midad entre profesor y el alumno, y la gestión de la influencia al enseñar. La proximidad se basa en el grado de cooperación y relación interpersonal que los profesores establecen con los alumnos, y la influencia refleja quién y cómo controla la comunicación en el aula. (p.210)

Según los resultados, se puede notar que existe una correlación significativa (moderada y positiva) entre la dimensión Seguimiento Académico y la variable DD, alcanzando $\mathrm{Rho}=0.507$ que equivale al $50.7 \%$ de explicación: permitiendo afirmar que si mejora la dimensión Seguimiento Académico entonces mejora la variable DD. Se refuerza esta evidencia con lo sostenido por Albione, Gregoret, Nuñez y Vitale (2005), sosteniendo:

La tutoría, además, favorece el acercamiento y relación entre docente y alumno. La barrera entre ambos se diluye, lo que permite, tanto a uno como a otro, hacer sugerencias y comentarios relativos a la materia de enseñanza, las actividades llevadas a cabo, cuestiones extracurriculares de índole formativa, etc. Asimismo, el carácter dialogante de la tutoría permite que el docente se acomode a las expectativas e intereses de los estudiantes, sus conocimientos previos, el grado de comprensión de la materia, etc. (p.8)

Finalmente, se puede afirmar que existe una correlación significativa (moderada y positiva) entre la dimensión Innovación y Aprendizaje y la variable DD, logrando Rho $=0.608$ que equivale al $60.8 \%$ de explicación: permitiendo afirmar que si mejora la dimensión Innovación y Aprendizaje entonces mejora la variable DD. Al respecto, Gros (2007) afirma que:

Un proceso innovador conlleva intención, planificación y esfuerzo pero puede fracasar en los resultados. Por ese motivo, la investigación es especialmente importante y relevante ya que posibilita elementos clave. Por un lado, dirigir los procesos de innovación a través del conocimiento básico generado por las investigaciones, y, por otro, evaluar y analizar los resultados de la innovación. (p.6)

\section{CONCLUSIONES}

- Con respecto a la correlación de variables, de acuerdo a los resultados y a la prueba de hipótesis realizada, se acepta la hipótesis general alterna $\left(\mathrm{HG}_{1}\right)$ y se rechaza la hipótesis general nula $\left(\mathrm{HG}_{0}\right)$, debido a que el $p$ obtenido $<p$ valor $(0.000<0.05)$. Además, el valor encontrado de $\mathrm{Rho}=0.629$ equivale 
al $62.9 \%$ de explicación de la variable DD por parte de la variable GA. Esta evidencia permite afirmar que la correlación es significativa (moderada y positiva) entre las variables GA y DD.

- En cuanto a la correlación entre la dimensión Diseño Curricular y la variable DD se observa que el $p$ obtenido $<p$ valor $(0.000<0.05)$, razón por la cual se acepta la hipótesis alterna $\left(\mathrm{H}_{1}\right)$ y se rechaza la hipótesis nula $\left(\mathrm{H}_{0}\right)$. Además, el valor encontrado de Rho $=0.527$ equivale al $52.7 \%$ de significancia, que es el nivel de dependencia de la variable DD con respecto a la dimensión Diseño Curricular.

- Existe una correlación significativa (aceptable y positiva) entre la dimensión Prácticas Pedagógicas y la variable DD, ya que se observa que el $p$ obtenido < p valor $(0.000<$ $0.05)$, razón por la cual se acepta la hipótesis alterna $\left(\mathrm{H}_{1}\right)$ y se rechaza la hipótesis nula $\left(\mathrm{H}_{0}\right)$. Además, el valor encontrado de Rho = 0.432 equivale al $43.2 \%$ de explicación, que es el nivel de dependencia de la variable DD con respecto a la dimensión Prácticas Pedagógicas.

- Se acepta la hipótesis alterna $\left(H_{1}\right)$ y se rechaza la hipótesis nula $\left(\mathrm{H}_{0}\right)$, con respecto a la correlación entre la dimensión Gestión de Aula y la variable DD, ya que se observa que el $p$ obtenido $<p$ valor $(0.000<0.05)$. Además, el valor encontrado de Rho $=0.601$ equivale al $60.1 \%$ de explicación.

- Con respecto a la correlación entre la dimensión Seguimiento Académico y la variable DD, se observa que el $p$ obtenido $<$ p valor $(0.000<0.05)$, razón por la cual se acepta la hipótesis alterna $\left(\mathrm{H}_{1}\right)$ y se rechaza la hipótesis nula $\left(\mathrm{H}_{0}\right)$. Además, el valor encontrado de Rho $=0.507$ equivale al $50.7 \%$ de explicación.

- Se acepta la hipótesis alterna $\left(\mathrm{H}_{1}\right)$ y se rechaza la hipótesis nula $\left(\mathrm{H}_{0}\right)$, referente a la correlación entre la dimensión Innovación y Aprendizaje con la variable DD que el $p$ obtenido $<p$ valor $(0.000<0.05$. Además, el valor encontrado de Rho $=0.608$ equivale al $60.8 \%$ de explicación.

\section{RECOMENDACIONES}

- Considerando que la GA es un criterio clave en la calidad de la enseñanza, es indispensable iniciar la capacitación del personal responsable de dicha gestión en la mencionada universidad con cursos de especialización relacionados a la gestión en instituciones educativas a nivel universitario. Todo esto con la finalidad de alcanzar elevados estándares de eficiencia y eficacia en relación a la gestión académica, ya que así se logrará optimizar el uso de los recursos y obtener las metas trazadas.

- Implementar talleres de capacitación docente en relación al coaching y liderazgo, a fin de elevar los estándares de comunicación hacia el alumnado. Si bien es cierto que el papel de la gestión académica es fundamental, lo es también la orientación por parte de los docentes desde una perspectiva actualizada que permita la formación integral de los estudiantes.

- A nivel de las prácticas pedagógicas es necesario fomentar en el personal docente la aplicación de la metodología educativa de las competencias docentes en la formación académica de los estudiantes y de esta forma se logrará que los ellos puedan alcanzar los desempeños adecuados para el desenvolvimiento profesional.

- En referencia al diseño curricular, se recomienda aplicar al detalle el aporte científico - educativo expuesto por la Dirección del Programa de Desarrollo Instruccional de la Universidad de Oklahoma(2003), donde se presenta seis elementos o clases de aprendizaje en relación al diseño de cursos universitarios, a saber: Conocimientos fundamentales, Aplicación, Integración, Dimensión humana, Compromiso y Aprendiendo cómo aprender.

- En cuanto al seguimiento académico es necesario diseñar estrategias orientadas en conseguir una mejor participación de los docentes en el proceso de seguimiento $y$ acompañamiento estudiantil. Asimismo, en la universidad un sistema eficaz de seguimiento de egresados, ya que el desempeño profesional y personal de los egresados permitirá determinar indicadores que logren medir la calidad y eficiencia de la institución educativa.

- Respecto a la innovación y aprendizaje, se sugiere: fomentar la producción académica del personal docente; incentivar la participación en la comunidad académica nacional e internacional del profesorado; coordinar a nivel de profesores la formación 
de grupos y líneas de investigación; y a nivel de estudiantes, establecer políticas de pasantía nacional e internacional así como motivar en el alumnado que sea consciente de que actualmente él es el verdadero protagonista en el aprendizaje y que el profesor cumple un rol de facilitador.

\section{AGRADECIMIENTO}

Se agradece la colaboración de las autoridades de la Universidad Privada Telesup, específicamente a los directivos de la Facultad de Ciencias Administrativas y Contables, por brindarnos el apoyo necesario para lograr la aplicación de los instrumentos de investigación en los diversos ambientes de dicha facultad. Por otra parte, agradecer a la comunidad estudiantil de la Facultad de Ciencias Administrativas y Contables, ya que sin su participación incondicional no se hubiese logrado los objetivos de la investigación. $Y$ finalmente, a los catedráticos de la plana docente especializada de la Facultad de Educación de la Universidad Nacional Mayor de San Marcos que permitieron dar la confiabilidad y validez de los instrumentos de investigación para el presente estudio científico.

\section{REFERENCIAS BIBLIOGRAFICAS}

[1] Albione, M., Gregoret, A., Nuñez, A. \& Vitale, B. (2005). Fortalecimiento del acompañamiento, seguimiento y control de los estudiantes en sus procesos académicos. V Coloquio Internacional sobre Gestión Universitaria en América del Sur. Universidad Nacional del Mar del Plata. Buenos Aires, Argentina.

[2] Almuiñas, J. \& Galarza, J. (2013). Prospectiva y evaluación del docente universitario. En A. Gonzáles (Ed.) La Evaluación del Desempeño del Docente Universitario. (pp. 29-46). Universidad Ecotec. Guayaquil, Ecuador.

[3] Alvarado, V., Cárcamo, A., García, A. \& Mella, E. (2008). Diseño Curricular basado en las competencias y Aseguramiento de la Calidad en la Educación Superior. Marco Conceptual Orientador para el Diseño Curricular Contemporáneo (pp.95-114). Centro Interuniversitario de Desarrollo-MINEDUC. Santiago de Chile, Chile.

[4] Association of American Schools in South America (2010). Manual OCSA / AASSA. Sistema de Evaluación del Desempeño de Docentes. USA: James H. Stronge.
[5] Correa, A., Correa, S. \& Álvarez, A. (2009). La gestión educativa, un nuevo paradigma. Fundación Universitaria Luis Amigó. Medellín, Colombia. Recuperado de: http://virtual. funlam.edu.co/repositorio/sites/default/ files/6lagestioneducativaunnuevparadigma.pdf

[6] De Escallón, I., Porter, G. \& Richler, D. (2013). Guía de indicadores de prácticas pedagógicas que favorecen la atención a la diversidad. Bogotá: Fundación Compartir.

[7] García, L. (2012). La Gestión académica: Retos, fundamentos y competencias. Bogotá: Editorial Kimpres Ltda.

[8] Gros, B. (2007). Tendencias actuales de la investigación en docencia universitaria. Edusfarm, revista d'educació superior en Farmàcia. (1),1-13. Recuperado de www. publicacions.ub.es/revistes/edusfarm1/ documentos/93.pdf

[9] Hernández, R., Fernández, C. \& Baptista, P. (2010). Metodología de la investigación. $5^{a}$ edición. México: Mc. Graw-Hill.

[10] Imbernon, F. (2009). Mejorar la enseñanza y el aprendizaje en la universidad. Barcelona: Ediciones Octaedro.

[11] Loureiro, S., Míguez, M. \& Otegui, X. (2016). Desempeño docente en la enseñanza universitaria: análisis de las opiniones estudiantiles. Cuadernos de Investigación Educativa, 7(1), 55-67 http://dx.doi. org/10.18861/cied.2016.7.1.2576

[12] Martín, X., Perdomo, I. \& Segredo, A. (2013). Capital humano, gestión académica y desarrollo organizacional. Educación Médica Superior, 27(3), 288-295. http://scielo.sld.cu

[13] Sánchez, J. \& Solar, M. (2007). Evaluación del Desempeño Docente y Calidad de la Docencia Universitaria. Modelo para la Evaluación del Desempeño Docente (pp.134-156). Centro Interuniversitario de Desarrollo-MINEDUC. Santiago de Chile, Chile.

[14] Torrego, J.C. (2008). El profesor como gestor del aula. En A. de la Herrán y J. Paredes (Coords.), Didáctica General. La práctica de la enseñanza en educación infantil, primaria y secundaria (pp.197-214). Madrid: McGraw-Hill.

[15] Universidad de Oklahoma. Dirección del Programa de Desarrollo Instruccional (2003). Una Guía Auto-Dirigida al Diseño de Cursos para el Aprendizaje Significativo. San Francisco, USA: Jossey-Bass. 